\title{
Communicating Dominance in a Nonanthropomorphic Robot Using Locomotion
}

\author{
JAMY LI, University of Twente \\ ANDREA CUADRA, BRIAN MOK, and BYRON REEVES, Stanford University \\ JOFISH KAYE, Mozilla \\ WENDY JU, Cornell University
}

\begin{abstract}
Dominance is a key aspect of interpersonal relationships. To what extent do nonverbal indicators related to dominance status translate to a nonanthropomorphic robot? An experiment $(N=25)$ addressed whether a mobile robot's motion style can influence people's perceptions of its status. Using concepts from improv theater literature, we developed two motion styles across three scenarios (robot makes lateral motions, approaches, and departs) to communicate a robot's dominance status through nonverbal expression. In agreement with the literature, participants described a motion style that was fast, in the foreground, and more animated as higher status than a motion style that was slow, in the periphery, and less animated. Participants used fewer negative emotion words to describe the robot with the purportedly high-status movements versus the purportedly low-status movements, but used more negative emotion words to describe the robot when it made departing motions that occurred in the same style. This result provides evidence that guidelines from improvisational theater for using nonverbal expression to perform interpersonal status can be applied to influence perception of a nonanthropomorphic robot's status, thus suggesting that useful models for more complicated behaviors might similarly be derived from performance literature and theory.
\end{abstract}

\section{CCS Concepts: • Human-centered computing $\rightarrow$ Empirical studies in interaction design;}

Additional Keywords and Phrases: Nonanthropomorphic robot, dominance, status, theater, motion path, human-robot interaction

\section{ACM Reference format:}

Jamy Li, Andrea Cuadra, Brian Mok, Byron Reeves, Jofish Kaye, and Wendy Ju. 2019. Communicating Dominance in a Nonanthropomorphic Robot Using Locomotion. ACM Trans. Hum.-Robot Interact. 8, 1, Article 4 (March 2019), 14 pages.

https://doi.org/10.1145/3310357

\footnotetext{
The majority of this work was performed when J. Li was at Stanford University.

The majority of this work was performed when J. Kaye was at Yahoo.

The majority of this work was performed when W. Ju was at Stanford University.

Authors' addresses: J. Li, University of Twente, P.O. Box 217, Enschede, OV 7500 AE, The Netherlands; email: j.j.li@ utwente.nl; A. Cuadra, Cornell University, 1 East Loop Rd, \#25H, New York, NY 10044, USA; email: apc75@cornell. edu; B. Mok, BMW, 2606 Bayshore Pkwy, Mountain View, CA 94043, USA; email: mahado@gmail.com; B. Reeves, Stanford University, 450 Serra Mall, Building 120, Room 110, Stanford, CA 94305, USA; email: reeves@stanford.edu; J. Kaye, Mozilla, 331 E Evelyn Ave, Mountain View, CA 94041, USA; email: jofish6@jofish.com; W. Ju, Cornell University, 1 East Loop Rd, \#25H, New York, NY 10044, USA; email: wendyju@cornell.edu.

Permission to make digital or hard copies of all or part of this work for personal or classroom use is granted without fee provided that copies are not made or distributed for profit or commercial advantage and that copies bear this notice and the full citation on the first page. Copyrights for components of this work owned by others than the author(s) must be honored. Abstracting with credit is permitted. To copy otherwise, or republish, to post on servers or to redistribute to lists, requires prior specific permission and/or a fee. Request permissions from permissions@acm.org.

(C) 2019 Copyright held by the owner/author(s). Publication rights licensed to ACM.

2573-9522/2019/03-ART4

https://doi.org/10.1145/3310357
}

ACM Transactions on Human-Robot Interaction, Vol. 8, No. 1, Article 4. Publication date: March 2019 


\section{INTRODUCTION}

An important aim of robotics for use in daily life is to create creatures capable of social expression [12]. Dominance is a key part of social expression in primates and other animals because it can be used to resolve conflicting goals or to place oneself in a social hierarchy. Researchers have explored how dominance can be perceived in anthropomorphic robots using dominance cues from interpersonal psychology, such as standing or relative height [25, 33]. However, broader guidelines on how to express dominance are missing in the human-robot interaction literature. Recent literature has indicated that guidelines and movement systems from dance and theater can be applied to nonanthropomorphic robot behavior to indicate mood or intent [23]. In Impro, Johnstone describes how to manipulate perceived status using motion: "the preferred position of a servant is usually at the edge of the master's 'parabola of space'... he invades the master's space 'unwillingly'... the servant has to be quiet, to move neatly... so that their bodies take up a minimum of space" [22, p. 64]. It would be useful to learn whether dominance behaviors used in improvisational theater can be applied to nonanthropomorphic robots to communicate status, which would allow human-robot interaction designers to draw on a larger potential body of performance knowledge in designing nonanthropomorphic robot behaviors.

Can a nonanthropomorphic robot's motion be used to communicate its status? Do principles of dominance status found in improvisational theater translate to a robot? The present study addressed these questions by comparing a robot motion path designed based on how Johnstone alleges that a "master" or a "servant" would move. The goal is to examine whether a robot's motion path could influence people's impressions of its dominance status, viewing dominance as a variable state rather than an inherent trait.

\section{BACKGROUND}

\subsection{Dominance in People and Robots}

Dominance refers to situation-dependent interaction patterns in which one person's assertion of control is met by another's acquiescence (cf. [10]). People are more satisfied when interacting with a partner who is complementary in dominance (e.g., dominant when they are subordinate) compared to similar in dominance [9]. People also adopt behavior that is complementary to the dominance of an interaction partner (e.g., acting dominant when their partner acts subordinate) [46]. Nonverbal indicators of dominance include high amount of eye contact, close proximity, and expansive body postures [2,21]. These indicators of interpersonal dominance have been used to design humanoid technology agents; for example, Isbister and Nass [18] varied the extraversion of a computer character's speech and gestures to make it similar or complementary in dominance to a person.

It is therefore unsurprising that past work on dominance in robots mostly conveys dominance using principles from interpersonal psychology. Li et al. [25] found that people disliked a humanoid robot when it spoke dominantly and used dominant posture compared to subordinate speech and posture. Groom et al. [15] found that people disliked a humanoid robot when it blamed its user for poor performance (an evaluative act that can be used to establish dominance) compared to when it did not. Roubroeks et al. [37] found that people experienced higher psychological reactance when a humanoid robot was dominant by giving highly threatening advice compared to nonthreatening advice. Rae et al. [33] found that people spoke more with a telepresence robot and had better impressions of themselves when the robot had a low height compared to a taller and more dominant height. Dautenhahn et al. [8] found that some people mentioned a front approach by a robot being more threatening compared to a side approach. Saerbeck and Bartneck [39] found that a robot accelerating quickly was more dominant than when it accelerated slowly. Past research on dominance in robots overlooks improv theater literature; yet, improv literature such as [22] can 
Table 1. Indicators of Dominant Motion Inspired by Improv Used in This Work

\begin{tabular}{|l|l|l|l|l|}
\hline Indicator & $\begin{array}{l}\text { Reference in } \\
\text { Johnstone [22] }\end{array}$ & $\begin{array}{l}\text { Reference in } \\
\text { Other Sources }\end{array}$ & $\begin{array}{l}\text { Purported High } \\
\text { Status (Motion A) }\end{array}$ & $\begin{array}{l}\text { Purported Low } \\
\text { Status (Motion B) }\end{array}$ \\
\hline Trajectory & $\begin{array}{l}\text { "master's } \\
\text { 'parabola of } \\
\text { space" (p. 64) }\end{array}$ & $\begin{array}{l}\text { Curvature only: } \\
\text { Saerbeck and } \\
\text { Bartneck [39] }\end{array}$ & $\begin{array}{l}\text { In front of person, } \\
\text { within 60 degrees }\end{array}$ & $\begin{array}{l}\text { To the side of } \\
\text { person, outside } \\
\text { 60 degrees }\end{array}$ \\
\hline Speed & $\begin{array}{l}\text { "Strolling" vs. } \\
\text { "hesitant" motion } \\
\text { (p. 59) }\end{array}$ & $\begin{array}{l}\text { Saerbeck and } \\
\text { Bartneck [39] }\end{array}$ & Fast speed, 0.4m/s & Slow speed, 0.2m/s \\
\hline Rpin rotation & $\begin{array}{l}\text { "Turn their backs } \\
\text { when they leave" } \\
\text { (p. 48) }\end{array}$ & n/a & Present & Absent \\
\hline $\begin{array}{l}\text { "straight" vs. } \\
\text { "shrink" (p. 37, } \\
\text { p. 64) }\end{array}$ & $\begin{array}{l}\text { Height only: Rae } \\
\text { et al. [33] }\end{array}$ & Present, 3 cm & Absent \\
\hline $\begin{array}{l}\text { "Expansive" vs. } \\
\text { "restrained" space } \\
\text { (p. 59) }\end{array}$ & Huang et al. [21] & Large & Small \\
\hline
\end{tabular}

give new insight into dominance in robots because it features a broad range of performative guidelines for gesture and locomotion, which is particularly important for nonanthropomorphic robots.

\subsection{Dominance in the Performing Arts Applied to Robot Design}

Dominance is recognized in improv literature as important for believable interaction between people. Improvisational theater uses gesture, locomotion, and speech to express an actor's dominance at a given moment. These "dominance status" behaviors [22] communicate an actor's inthe-moment state rather than an inherent trait. Dominance status is separate from social status because characters can pretend to be higher or lower status than their actual social standing [38]. Improvisational theater teaches strategies to create believable motions rather than specifying how to behave in a scene [44]. Facing a person, moving in front of the individual, moving freely in a space, and approaching a person without hesitation are high-status behaviors used by actors [22, p. 59]. Table 1 shows how these principles offer a unique perspective on the generation of dominance that differs from psychology-based works: for example, Johnstone defines a parabolic area as important for trajectory, whereas Saerbeck and Bartneck [39] look only at curvature of motion. Improv principles use the same variables as past work on dominance in robots, but manipulate them in a different way.

Many other concepts and techniques from the performing arts have already been successfully applied to creating new expressions in robots. Laban motion analysis is a theater technique that groups human motion into key components (e.g., space, effort) that are perceivable by audiences and useful for performers [30]. Knight and Simmons [23] asked experts trained in Laban techniques to create emotional motions for a nonanthropomorphic robot, which they used to train machinelearning algorithms. Participants who saw 2D graphical renderings of the robot motion were able to judge better than chance which of six different manners (e.g., shy) each represented. Butler and Agah [4] also used Laban to design three approaches for a robot. They found that participants rated a $40 \mathrm{in} / \mathrm{sec}$ frontal approach by a robot to be very unpleasant compared to a $10 \mathrm{in} / \mathrm{sec}$ frontal or a $20 \mathrm{in} / \mathrm{sec}$ side approach, particularly when the robot had a tall humanoid body versus when it had only a short base. They also found that people preferred a robot to pass by with motion that did not stop rather than motion that did. Sharma et al. [41] had an artist design motion paths for a quadcopter based on the Laban effort system. Motions that were meandering and quick were 
perceived as having more positive valence than those that were direct and slow. Motions that were meandering, strong, quick, and curvy were perceived as having higher arousal than those that were direct, light, slow, and straight. Lourens et al. [27] compared Laban experts' analysis of the hand motions of actors expressing emotions with acceleration profiles and argued that Laban could serve as a common language for robots and humans. Apart from the Laban system, Takayama et al. [45] used character animation to design robot motion and found that robot gaze and head motion improved participants' ratings of forethought in the robot. Improvisational theater experts designed movements for the same robot used in the current work [19] but evaluated the effect of the resulting movements on the percentage of people who placed their feet on the robot and qualitative themes [42] rather than people's perception of dominance status. We note that these works mostly use Laban analysis and have not explored whether their techniques can convey dominance in a robot. Given past successes in applying animation and Laban theater techniques to robots, we hypothesize that improvisational theater techniques that actors use to convey dominance will be similarly effective for robots.

Hypothesis 1: Purported high-status robot motion will be perceived as higher dominance status than purported low-status robot motion.

Apart from a description of how to communicate dominance, improv literature also describes the effect of dominance on an audience. Dominance is a "see-saw" [22]: one person goes up when the other goes down. Thus, we also investigate whether robot motion designed using theater techniques can elicit feelings of relational dominance or acquiescence in a person.

Hypothesis 2: Purported high-status robot motion will be perceived as dominating its user more than purported low-status robot motion.

Moreover, people may cheer up when a robot lowers its dominance because they rise by contrast-unless they identify with the robot and "sit on [its] end of the see-saw" [22]. We therefore also test whether dominance behaviors from improv theater affect people's emotional responses.

Research Question: Will purported high-status robot motion receive comments that are higher or lower in emotional tone than purported low-status robot motion?

We note that a variety of techniques from areas of the performing arts could be used to design dominance in a robot. For example, Chekhov's "psychological gesture" condenses the state of a character into a single motion that can be shown to the audience or imagined in the actor's mind to guide a performance [6]; for example, a large upward motion of the arms to represent a dominant personality. Grotowski's "poor theater" focuses on actors' kinesthetic learning by physically moving and manipulating their bodies [16], which could lead to motion that affects physiological dominance more than verbal explanation. Biomechanics is a method of teaching actors how the body executes physical reflexes to external stimuli [14] that might also be used to guide the design of dominance. A non-improv actor handbook (e.g., [38, p. 84]) mentions that high dominance status can be achieved by showing people one's self-improvement, which is difficult for a robot. We focus on improv theater literature [22] because it addresses dominance more explicitly than the above work.

\subsection{Design and Communicativeness of Nonanthropomorphic Robot Motion}

Performing arts' guidelines for gesture and locomotion may be a particularly valuable source of inspiration for nonanthropomorphic robots that are not capable of speech. These "RObjects" [11, 47] are everyday objects fitted with wheels. Examples include chairs that park themselves after a meeting [1]; benches that automatically move down a queue while their users stay seated [28]; ottomans that place themselves in front of a user's feet [42]; trash cans that drive to passersby [49, 
50]; and storage boxes that move toward clutter to teach children to clean their rooms [11]. Since performing artists may be accustomed to using simple motions to establish relational narratives, their principles may work with "abstract robots" in which the artifact embodies only a core abstract concept to tap into observers' diverse experience of that concept.

Past work on nonanthropomorphic robots has found that their motion can be highly expressive but has not looked at whether their motion can be designed to effectively express dominance. Cauchard et al. [5] used aesthetics to design motions for three roles of a flying drone (an adventurer was represented with high speed, spins, and flips; anti-social was represented with moderate speed and brief stops; and exhausted was represented with slow speed and wobbles). They found that people could identify the correct emotions corresponding to each role (correctness determined by a pretest without the robot) and the drone's intent (e.g., take photo) better than chance. Löffler et al. [26] also used aesthetics to design fast rotations and circular motion in a ground robot to express joy, slow rotations away from the user for sadness, jumpy movements away from the user for fear, and shaking movements toward the user for anger. They found that fear is best communicated by motion, joy is best communicated by sound plus motion, and other emotions are best communicated by color and sound. Song and Yamada [43] used human-computer interaction literature to design high vibration for joy, low vibration for sadness and very high vibration for anger in a robot. They found that participants are able to identify which of four emotions a vibrating robot communicates better than chance. Yamaji et al. [49] did not specify the method that they used to design motion and found that a robotic trashcan can better communicate its intent to collect garbage by moving toward garbage as opposed to moving toward a user; people also picked up garbage more frequently when the robot moved toward the garbage compared to when it moved toward the person. Young et al. [52] used participants to puppet a robot's motion to express various roles (e.g., stalker) and used resulting data to train machine-learning algorithms capable of generating those roles. They found that participants could identify the role when the motions were played back to them in a random order. Walker et al. [48] looked at mixed-reality literature to create different motion indicators for a drone robot. They found that a flying drone's intent was clearer when it used augmented reality markers than when it did not but did not explore variations in motion alone. Fink et al. [11] designed a robot's motion to be proactive or reactive and found that children explored more with a proactive robotic box that moved around a room while wiggling and tidied up more with a reactive robotic box that wiggled only after toys were put inside but did not evaluate how children perceived the robot. Pacchierotti et al. [31] used proxemics to design motions for a robot that passes people in a hallway. They found that people preferred when the robot moves to the side earlier rather than later and quickly rather than slowly. Given these works demonstrating that people are able to recognize emotion and proactiveness through motion of a nonanthropomorphic robot plus other work demonstrating that people can identify signs of dominance in the motion of 2D shapes on a screen [17], we use a nonanthropomorphic robot in the current research as a test of a lower bound for perception of dominance in robots.

\section{CURRENT STUDY}

The present work evaluates whether motion style can influence people's perception of a nonanthropomorphic robot's dominance. We test whether this effect can be achieved through motion designed using principles from improv theater about the expression of dominance in interpersonal communication. We hypothesize that people will perceive purported high-status motion as higher-dominance status and higher relational dominance than purported low-status motion. We also explore whether people prefer purported high-status or low-status motions for three usage scenarios of a robot footstool to obtain scenario-specific design recommendations. 


\section{MATERIALS AND METHODS}

\subsection{Participants}

Twenty-five English-speaking participants (16 female, 9 male) affiliated with Stanford University between the ages of 19 and 46 (mean $[M]=21.4$, standard deviation $[S D]=5.1$ ) received $\$ 20$ for their participation. The Human Subjects Research Board at Stanford University approved the study.

\subsection{Study Design}

A 2 (motion style: purported high-status motion vs. purported low-status motion) $\times 3$ (scenario: lateral movement, approach vs. depart) within-participants experiment was conducted to investigate the effect of two different improv-inspired motion styles on people's perception of a robot's status. Motion styles were designed using concepts from improvised theater [19, 22] (see Table 1) by a member of the research team.

\subsection{Procedure}

The study took place in a university laboratory room set up as a living room. After granting consent, participants were asked to sit in a chair and were instructed as follows: "Please pretend that you are at home by yourself in your living room and relaxing. You are browsing the Internet on the laptop in front of you. The robot is elsewhere in the room, in the 'background.' You aren't specifically watching it." This sitting and observing procedure was used in past studies in dominance with robots $[8,33,39]$, perhaps because it controls for participant location. Participants then saw six trials: the three scenarios for either purported high-status or purported low-status motion (depending on random assignment), then the three scenarios for the other motion style. Each trial consisted of four phases. In the waiting stage, the participant browsed the Internet. In the motion phase, the robot executed a movement. In the interview stage, the participant was asked open-ended questions by a human interviewer sitting at the other end of the room (beginning with "How would you describe the robot's movement?" with prompts "How would you describe its personality if you think it had any?" and "How much, if at all, do you feel like the robot is relating to you?"). In the survey stage, the participant answered two questions on perceived relational dominance ("The robot tried to dominate me" and "The robot tried to control the interaction") [3]. The experiment lasted 30 minutes.

\subsection{Materials}

The robotic ottoman is a $0.5 \mathrm{~m}$ tall $^{1}$, cube-shaped, 3-degrees-of-freedom robot footstool (Figure 1; from [42]). The robot was able to move forward or backward, rotate, and lift itself up or down. It was built using an iRobot Create with a dark brown polyurethane leather-like covering.

\subsection{Wizard of $\mathrm{Oz}$}

A hidden remote operator ("Wizard of Oz") controlled the robot using a live video feed of the study room and a game controller wirelessly connected to the robot. The same wizard ran all sessions. This method was used to support user evaluation of a design prototype prior to full autonomy and technical constraints [7]. The wizard had 1 month of experience using the robot and 3 days of training for the specific motions outlined in the present study. Training was focused on ensuring that the purported high-status and low-status motions appeared as outlined in Table 1. Although reproducing perfectly identical motion was difficult owing to limitations in the robot's mechanical system and floor traction, training sessions ensured relatively consistent behavior (as recommended by [36]).

\footnotetext{
${ }^{1}$ The robot is $41 \%$ of the average male and $45 \%$ of the average female sitting eye height for U.S. adults.
} 


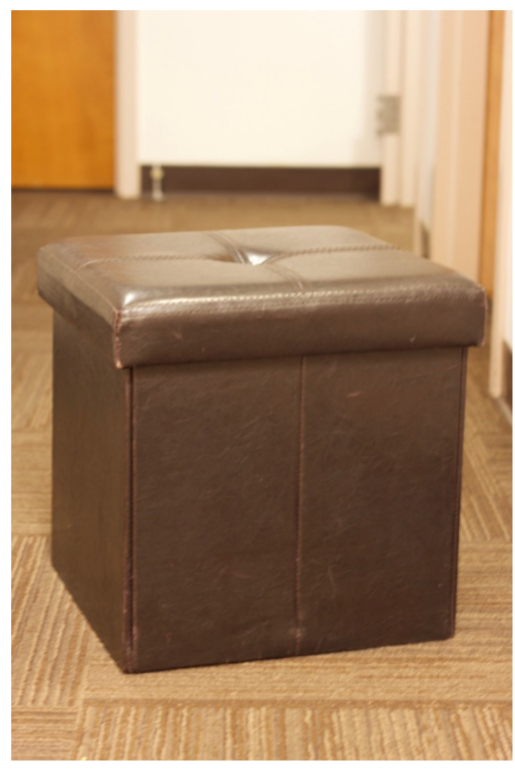

Fig. 1. Photo of robot footstool.

\subsection{Analysis}

Interview responses were categorized into high-, neutral-, and low-status perception of the robot using directed qualitative content analysis [20, 29, 40, p. 170]. Three independent researchers blind to condition read through a randomized list of interview transcripts, marking all text that described the robot's status. Two 1-hour collaborative sessions at the third-way point resulted in a common code book (Table 2). Perceived status of a given trial was the average score given by the three coders [35, p. 298]. Interrater reliability (Fleiss's Kappa) was 0.651, a value interpreted as substantial agreement [24].

Emotional tone was calculated using LIWC $^{2}$ [32] as the number of negative emotion words divided by the total number of words, since negative emotion words were stronger than positive ones in prior work [8].

We compared the ratings given by the participants who saw purported high-status motion first with those who saw purported low-status motion first to assess carryover effects in our repeatedmeasures study design. There were no significant differences between the orders for perceived dominance status, $t(21)=0.78, p=0.45$, or perceived relational dominance, $t(22)=1.2, p=0.23$.

All statistical analyses were done using $\mathrm{R}$ version 3.4.2 and RStudio.

\section{RESULTS}

\subsection{Perceived Dominance Status}

Repeated-measures analysis of variance (ANOVA) ${ }^{3}$ revealed a significant main effect of motion style on perceived dominance status (Figure 2), $F_{1,17}=5.7, p=0.029, \eta_{\mathrm{p}}{ }^{2}=0.25$. Participants spoke

\footnotetext{
${ }^{2}$ http://liwc.wpengine.com.

${ }^{3}$ Modeled as status $\sim$ motion style * scenario + Error (participant/(motion style * scenario)). Analyses were repeated using lmer mixed-effects modeling and yielded similar results. Partial eta squared effect sizes were estimated as the sum of squares of the effect divided by that sum of squares plus the residual sum of squares.
} 
Table 2. Code Book for Perceived Status Dominance

\begin{tabular}{|c|c|c|c|}
\hline Value & Meaning & Indicators & Example \\
\hline+1 & $\begin{array}{l}\text { Robot shows } \\
\text { high status. }\end{array}$ & $\begin{array}{l}\text { Confident, strong, decisive, } \\
\text { standoffish, dominant, } \\
\text { obedient w/ determined }^{a}\end{array}$ & $\begin{array}{l}\text { "Reversing or just kind of turned away, } \\
\text { immediately drove away from me and kind } \\
\text { of got to its point and then went over back to } \\
\text { the spot by the wall. So maybe a little } \\
\text { dismissive or like ... Yeah, I guess that's like } \\
\text {... That's kind of ... Felt like maybe it was } \\
\text { dissatisfied." (+1, all } 3 \text { coders) }\end{array}$ \\
\hline 0 & $\begin{array}{l}\text { Robot shows } \\
\text { neutral status. }\end{array}$ & $\begin{array}{l}\text { Equal to me }{ }^{a} \text { (no } \\
\text { status-related comments) }\end{array}$ & $\begin{array}{l}\text { "Some movement is a little bit small back } \\
\text { and forward. I feel like its personality is } \\
\text { scared or timid, shy. It's relating to me in a } \\
\text { way there, even though it's shy, it's also } \\
\text { willing to put theirselves [sic] out there. If } \\
\text { people were to tell it to do something, it } \\
\text { wouldn't. In some ways, it could also be } \\
\text { disobedient." (0, all } 3 \text { coders) }\end{array}$ \\
\hline-1 & $\begin{array}{l}\text { Robot shows } \\
\text { low status. }\end{array}$ & $\begin{array}{l}\text { Not confident, nervous, } \\
\text { anxious, shy, indecisive, } \\
\text { servant, confused }^{a} \text {, obedient } \\
\text { w/o determined }^{a}\end{array}$ & $\begin{array}{l}\text { "Obedient, ah. A dog. Happy." ( }-1, \\
\text { all } 3 \text { coders) }\end{array}$ \\
\hline
\end{tabular}

${ }^{a}$ Obedience was coded as the robot having low status, except when paired with "determined," in which case two researchers coded as low status and one coded as high status. "Equal to me" was neutral unless paired with high status indicators, in which case it was coded as high status. "Confused" was coded as low status only if it was accompanied by other words suggesting low status.

Interrater reliability among 3 coders was Fleiss's $\kappa=0.651$. Indicators are words participants used that were judged to be related to status.

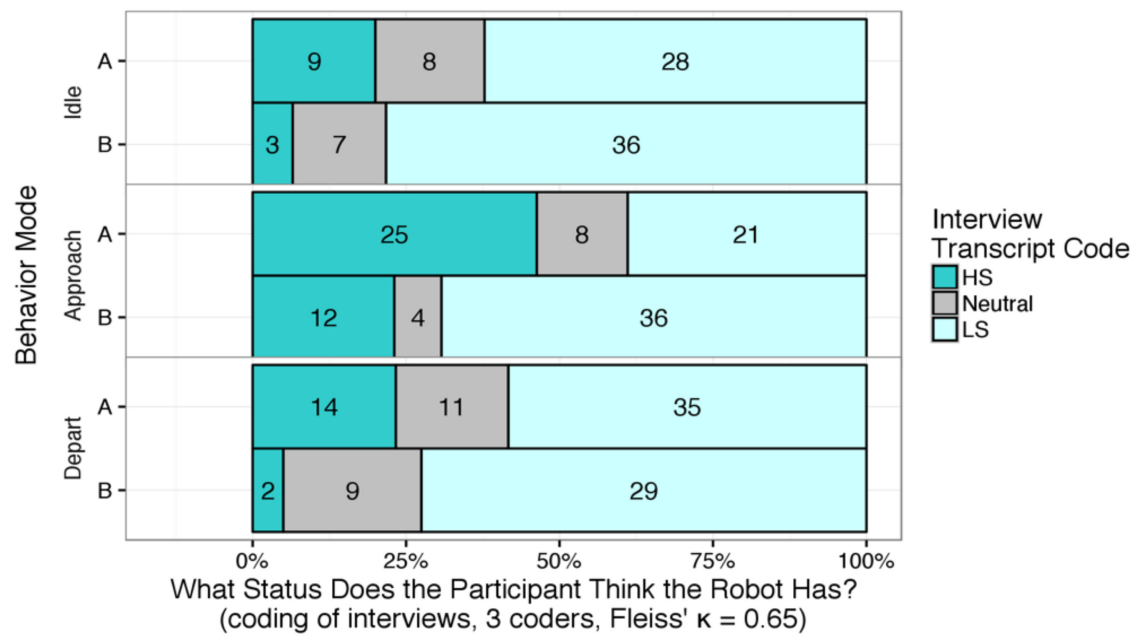

Fig. 2. Horizontal proportion plot of the effect of motion path on interview transcript codes by behavior mode. Participants spoke of the robot as being higher status with Motion A (purported high status) than with Motion B (purported low status). Numbers give the total counts from all coders, excluding responses that were given no code. 


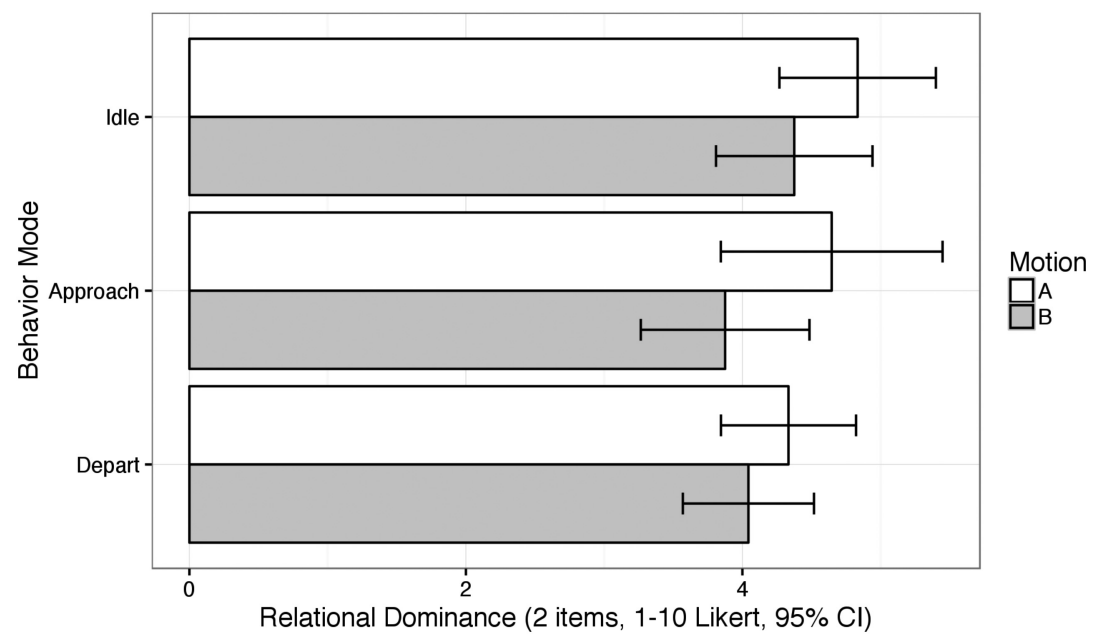

Fig. 3. Horizontal bar plot of the effect of motion path on perceived relational dominance by behavior mode. Motion path was purported high status (Motion A) or purported low status (Motion B). Error bars show $95 \%$ $\mathrm{Cl}$.

of the robot as being higher status (see Table 2) with purported high-status motion than purported low status motion, $M=-0.24, S D=1.0$ vs. $M=-0.54, S D=0.8,95 \%$ confidence interval (CI) 0.04 $<\mu_{\text {motion style A }}-\mu_{\text {motion style B }}<0.57$. No main effect of scenario, $F_{2,38}=2.6, p=0.09, \eta_{\mathrm{p}}{ }^{2}=0.12$, or interaction effect was found, $F_{2,32}=0.1, p=0.91, \eta_{\mathrm{p}}{ }^{2}=0.01$. Hypothesis 1 was supported.

\subsection{Perceived Relational Dominance}

Repeated measures ANOVA revealed no significant main or interaction effects on perceived relational dominance (Figure 3). No significant differences were found in the perception of the robot's relational dominance for motion style, $F_{1,22}=2.6, p=0.12, \eta_{\mathrm{p}}{ }^{2}=0.11$, scenario, $F_{2,45}=1.2, p=0.32$, $\eta_{\mathrm{p}}{ }^{2}=0.05$, or their interaction, $F_{2,45}=0.5, p=0.61, \eta_{\mathrm{p}}{ }^{2}=0.02$. Hypothesis 2 was not supported.

\subsection{Emotional Tone}

Repeated-measures ANOVA revealed a significant motion style $\times$ scenario interaction for emotional tone (Figure 4): $F_{2,43}=4.8, p=0.013, \eta_{\mathrm{p}}{ }^{2}=0.18$. Participants spoke less negatively when the robot used purported high-status versus purported low-status motion in the lateral movement scenario but spoke more negatively about a robot that used motion that occurred in the same style in the departing scenario. This addresses the research question by finding that people's preference for a high- versus low-status robot is based on the scenario.

Participants spoke about purported high-status motion in the lateral scenario as being active, e.g., "I have a dog... sometimes I'm aware that they're sort of cruising around at about this range, like doing their own thing, but I have a little bit of like, 'Oh. I wonder what they're up to.' There was a hint of that... with this guy." Conversely, participants spoke negatively about purported low-status motion in the lateral scenario because the robot did not demonstrate greater movement capability or felt "restless." Participants seeing purported high-status motion in the depart scenario said the robot appeared "offended" or "annoyed." Participants seeing purported low-status motion in the depart scenario said that the robot appeared to be "shy" or "obedient." 


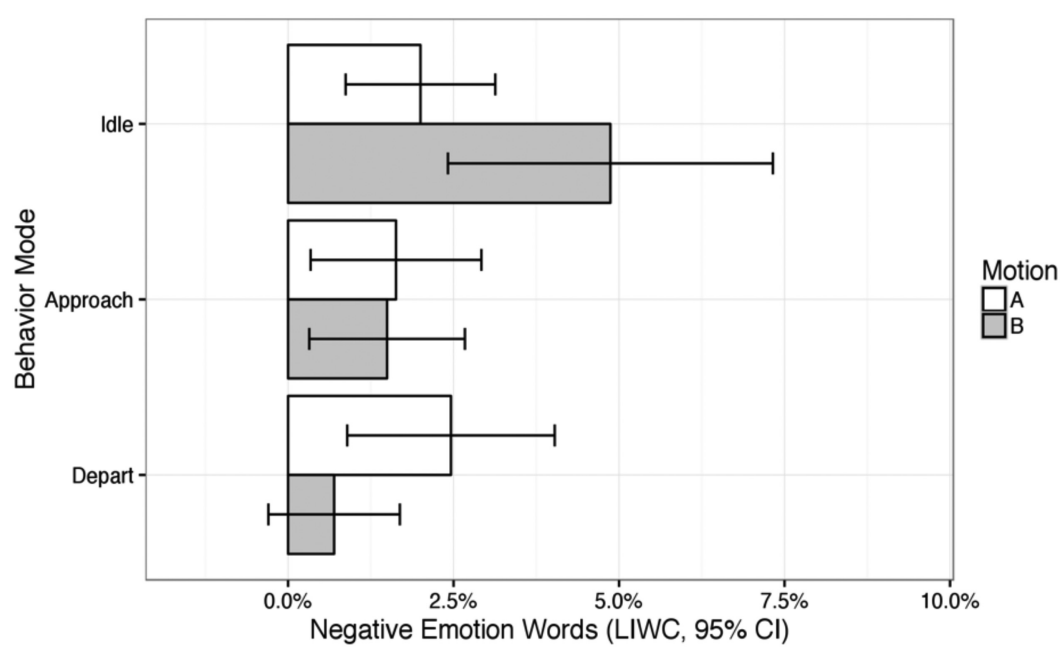

Fig. 4. Horizontal bar plot of the effect of motion path on emotional tone by behavior mode. A robot footstool was perceived more positively with purported high-status behavior while idling but was perceived less positively with purported high-status behavior while departing. Error bars show $95 \% \mathrm{Cl}$.

\section{DISCUSSION}

\subsection{Overview of Results}

An abstract, nonanthropomorphic robot's motion path can influence how people perceive its dominance status. People perceived a robot that moved with purported high status as higher status than one that moved with purported low status. In addition, a robot that moved with purported high status was liked more than one that moved with purported low status when it was making lateral movements unrelated to the person, but people felt the opposite when it was departing from them.

\subsection{Theoretical Implications}

The motion paths that human actors use to convey interpersonal dominance can also make an abstract, nonanthropomorphic robot seem dominant. This is because we inherently see and respond to social cues in locomotion, even when those cues come from a robot instead of a person $[13,17,34]$. This is in spite of the fact that the robot used in the current study did not have a human morphology, a human-like face, or markings indicating it had a front side to it. Perhaps most important, our finding demonstrates that improv literature can be used to extend the design space of robot motion expressivity to include status. Expressing status is particularly attractive for robots with limited capabilities because it might be reliable for observers to identify, since its categorization into high and low dominance is more constrained than emotion (e.g., valence and arousal, each of which can be high and low) or intent (e.g., one of a multitude of possible intents).

\subsection{Design Implications}

Designers can apply status behaviors that actors use to a robot to influence the robot's perceived status. Purported high-status motion (fast speed, in front of a person, with lifts) can make a nonanthropomorphic robot appear higher status than purported low-status motion (low speed, to the side of a person, without lifts). Designers can shift from purported high-status to low-status motion to suit the robot's current situation. Whether people prefer a nonanthropomorphic robot to 
have high versus low status depends on context. In a departing scenario, people prefer a robot that moves with low status, possibly because people view themselves as high status and prefer complementary behavior. In a lateral-motion scenario, people prefer a robot that moves with high status, perhaps because it is not interacting with them.

\subsection{Limitations and Future Work}

Can motion paths using purported high- and low-status motion from improvisational theater literature make autonomous vehicles, security bots, or other objects appear high or low status? This is an open question since we did not test multiple robotic forms. Improv literature recommends that actors be given context to arrive at their own design choices [44]; similarly, we presented a general design method of using literature from the performing arts to design a robot's motions to suit its context. A larger robot using the same motion paths as the current robot may lead greater intimidation and dislike with a dominant approach than the robot in this study (cf. [8]). A stationary robot with manipulators would also need different motions.

Participants felt that the robot was acting dominant with purported high-status motion, but not that it was dominating them. This may be a limitation of the within-participant design. Since relational dominance was measured using survey items after each robot behavior was presented, participants may have thought more thoroughly about whether a footstool could be dominant than in a between-participant design.

In our study design, the robot had only one role (i.e., to serve the user). We expect that dominance status behaviors would be more interpretable in a robot that switches between high-and low-status tasks-for example, an ottoman that is subordinate when it approaches its user's feet but dominant when it pushes away an intruder. Our study also took place in a laboratory, whereas conducting the study in participants' homes or with a realistic secondary task (e.g., watching a video on a laptop) could add to its external validity. We assessed people's perceptions of robot motion, but it would be interesting to test whether purported high-status or low-status motions influence how people behave with robots as future work.

We also note that the variables that we chose to manipulate (such as trajectory and speed) have been used for other concepts apart from dominance, such as affect (e.g., by Yoshioka et al. [51]). This may be a potential confounder and challenge in studying dominance in robots, since there is not yet an "established" manipulation of dominance for nonanthropomorphic robots. It is possible that affect, extraversion, or another characteristic was a mediator for people's perception of dominance in our study. Although we tried to address this by including open-ended questions, future studies could use generative coding methods or explore other perceived characteristics in the robot alongside dominance to establish a more detailed understanding of how people perceive the robot's motion.

\section{CONCLUSIONS}

We find that the purported movement patterns alleged by Johnstone [22] to indicate status do, in fact, seem to affect user perception of a robot as we would expect them to. Participants judged two motion styles with different dominance behaviors varying in speed, trajectory, and presence of spins. They perceived a nonanthropomorphic robot that used purported high-status motion as higher status than when it used purported low-status motion. The motion of a robot that moves independently in people's homes can influence how people perceive its dominance status, which might help to shape people's expectations and behaviors. This suggests that models for more complicated robot behaviors may profitably be drawn from theatrical performance literature and theory. 


\section{ACKNOWLEDGMENTS}

The authors thank Professor Pamela Hinds for use of the university laboratory room.

\section{REFERENCES}

[1] Hilary Brueck. 2016. Watch Nissan's new self-driving office chair in action. Retrieved October 1, 2016 from http:// fortune.com/2016/02/17/nissan-self-driving-chair/.

[2] Judee K. Burgoon, David B. Buller, Jerold L. Hale, and Mark A. de Turck. 1984. Relational messages associated with nonverbal behaviors. Hum. Commun. Res. 10, 3 (1984), 351-378.

[3] Judee K. Burgoon and Jerold L. Hale. 1987. Validation and measurement of the fundamental themes of relational communication. Commun. Monogr. 54, 1 (1987), 19-41. https://doi.org/10.1080/03637758709390214

[4] John Travis Butler and Arvin Agah. 2001. Psychological effects of behavior patterns of a mobile personal robot. Auton. Robot. 10, 2 (2001), 185-202.

[5] Jessica Cauchard, Kevin Zhai, Marco Spadafora, and James Landay. 2016. Emotion encoding in human-drone interaction. In Proceedings of the 11th ACM/IEEE International Conference on Human-Robot Interaction (HRI'16). IEEE, Los Alamitos, CA, 263-270. https://doi.org/10.1109/HRI.2016.7451761

[6] Franc Chamberlain. 2012. Michael Chekhov on the technique of acting: 'Was Don Quixote true to life?' In TwentiethCentury Actor Training, Alison Hodge (Ed.). Routledge, London, 97-115.

[7] Nils Dahlbac̈k, Arne Jon̈sson, and Lars Ahrenberg. 1993. Wizard of Oz studies-why and how. Knowl.-Based Syst. 6 , 4 (1993), 258-266.

[8] Kerstin Dautenhahn, Michael Walters, Sarah Woods, Kheng Lee Koay, Chrystopher L. Nehaniv, A. Sisbot, Rachid Alami, and Thierry Siméon. 2006. How may I serve you?: A robot companion approaching a seated person in a helping context. In Proceedings of the 1st ACM SIGCHI/SIGART Conference on Human-Robot Interaction (HRI'06). ACM, New York, NY, 172-179.

[9] D. Christopher Dryer and Leonard M. Horowitz. 1997. When do opposites attract? Interpersonal complementarity versus similarity. F. Pers. Soc. Psychol. 72, 3 (1997), 592-603.

[10] Norah E. Dunbar and Judee K. Burgoon. 2005. Perceptions of power and interactional dominance in interpersonal relationships. F. Pers. Soc. Psychol. 22, 2 (2005), 207-233.

[11] Julia Fink, Séverin Lemaignan, Pierre Dillenbourg, Philippe Rétornaz, Florian Vaussard, Alain Berthoud, Francesco Mondada, Florian Wille, and Karmen Franinović. 2014. Which robot behavior can motivate children to tidy up their toys?: Design and evaluation of Ranger. In Proceedings of the 2014 ACM/IEEE International Conference on Human-Robot Interaction (HRI'14). ACM, New York, NY, 439-446. https://doi.org/10.1145/2559636.2559659

[12] Terrence Fong, Illah Nourbakhsh, and Kerstin Dautenhahn. 2003. A survey of socially interactive robots. Robot. Auton. Syst. 42, 3-4 (2003), 143-166. https://doi.org/10.1016/S0921-8890(02)00372-X

[13] Jodi Forlizzi and Carl DiSalvo. 2006. Service robots in the domestic environment: A study of the Roomba vacuum in the home. In Proceedings of the 1st ACM SIGCHI/SIGART Conference on Human-Robot Interaction (HRI'06). ACM, New York, NY, 258-265.

[14] Nikolai Gorchakov. 1957. The Theater in Soviet Russia. Columbia University Press, New York, NY.

[15] Victoria Groom, Jimmy Chen, Theresa Johnson, F. Arda Kara, and Clifford Nass. 2010. Critic, compatriot, or chump? Responses to robot blame attribution. In Proceedings of the 5th ACM/IEEE International Conference on Human-Robot Interaction (HRI'10). IEEE Press, Piscataway, NJ, 211-218.

[16] Jerzy Grotowski. 2012. Towards a Poor Theatre. Routledge, New York, NY.

[17] Fritz Heider and Marianne Simmel. 1944. An experimental study of apparent behavior. Am. J. Psychol. 57, 2 (1944), 243-259.

[18] Katherine Isbister and Clifford Nass. 2000. Consistency of personality in interactive characters: verbal cues, nonverbal cues, and user characteristics. Int. f. Hum.-Comput. Int. 53, 2 (2000), 251-267.

[19] Guy Hoffman and Wendy Ju. 2014. Designing robots with movement in mind. f. Hum.-Robot Interact. 3, 1 (2014), 89-122. https://doi.org/10.5898/JHRI.3.1.Hoffman

[20] Hsiu-Fang Hsieh and Sarah E. Shannon. 2005. Three approaches to qualitative content analysis. Qual. Health Res. 15, 9 (2005), 1277-1288.

[21] Li Huang, Adam Galinsky, Deborah Gruenfeld, and Lucia Guillory. 2011. Powerful postures versus powerful roles which is the proximate correlate of thought and behavior? Psychol. Sci. 22, 1 (2011), 95-102. https://doi.org/10.1177\% 2F0956797610391912

[22] Keith Johnstone. 1979. Impro: Improvisation and the Theatre. Routledge, New York, NY.

[23] Heather Knight and Reid Simmons. 2014. Expressive motion with x, y and theta: Laban effort features for mobile robots. In Proceedings of the 23rd IEEE International Symposium on Robot and Human Interactive Communication (ROMAN'14). IEEE, Piscataway, NJ, 267-273. https://doi.org/10.1109/ROMAN.2014.6926264 
[24] J. Richard Landis and Gary Koch. 1977. The measurement of observer agreement for categorical data. Biometrics. 33, 1 (1977), 159-174.

[25] Jamy Li, Wendy Ju, and Clifford Nass. 2015. Observer perception of dominance and mirroring behavior in humanrobot relationships. In Proceedings of the 10th Annual ACM/IEEE International Conference on Human-Robot Interaction (HRI'15). ACM, New York, NY, 133-140. https://doi.org/10.1145/2696454.2696459

[26] Diana Löffler, Nina Schmidt, and Robert Tscharn. 2018. Multimodal expression of artificial emotion in social robots using color, motion and sound. In Proceedings of the 2018 ACM/IEEE International Conference on Human-Robot Interaction (HRI'18). ACM, New York, NY, 334-343. https://doi.org/10.1145/3171221.3171261

[27] Tino Lourens, Roos van Berkel, and Emilia Barakova. 2010. Communicating emotions and mental states to robots in a real time parallel framework using Laban movement analysis. Robot. Auton. Syst. 58, 12 (2010), 1256-1265. https:// doi.org/10.1016/j.robot.2010.08.006

[28] Cara McGoogan. 2016. Too lazy to queue? Nissan's robotic chairs are replacing lines in Japan. Retrieved October 10, 2016 from http://www.telegraph.co.uk/technology/2016/09/28/too-lazy-to-queue-nissans-robotic-chairsare-replacing-lines-in/.

[29] Francesca Moretti, Lisbeth van Vliet, Jozien Bensing, Guiseppe Deledda, Mariangela Mazzi, Michela Rimondini, Christa Zimmermann, and Ian Fletcher. 2011. A standardized approach to qualitative content analysis of focus group discussions from different countries. Patient Educ. Couns. 82, 3 (2011), 420-428.

[30] Jean Newlove and John Dalby. 2004. Laban for All. Taylor \& Francis, Burlington, MA.

[31] Elena Pacchierotti, Henrik I. Christensen, and Patric Jensfelt. 2005. Embodied social interaction in hallway settings: A user study. In Proceedings of the IEEE Workshop on Robot and Human Interactive Communication (RO-MAN'05). IEEE Press, Piscataway, NJ, 164-171.

[32] James W. Pennebaker and Martha E. Francis. 1999. Linguistic Inquiry and Word Count (LIWC). Lawrence Erlbaum Associates, Mahwah, NJ.

[33] Irene Rae, Leila Takayama, and Bilge Mutlu. 2013. The influence of height in robot-mediated communication. In Proceedings of the 8th ACM/IEEE International Conference on Human-Robot Interaction (HRI'13). IEEE Press, Piscataway, NJ, 1-8.

[34] Byron Reeves and Clifford Nass. 1996. The Media Equation: How People Treat Computers, Television, and New Media Like Real People and Places. Cambridge University Press, Cambridge, UK.

[35] Harry T. Reis and Charles M. Judd. 2000. Handbook of Research Methods in Social and Personality Psychology. Cambridge University Press, Cambridge, UK.

[36] Laurel Riek. 2012. Wizard of Oz studies in HRI: A systematic review and new reporting guidelines. F. Hum.-Robot Interact. 1, 1 (2012), 119-136.

[37] M. A. J. Roubroeks, J. R. C. Ham, and C. J. H. Midden. 2010. The dominant robot: Threatening robots cause psychological reactance, especially when they have incongruent goals. In International Conference on Persuasive Technology (PERSUASIVE'10). Springer, Berlin, 174-184. https://doi.org/10.1007/978-3-642-13226-1_18.

[38] John Rudlin. 1994. Commedia Dell'arte: An Actor's Handbook. Routledge, London.

[39] Martin Saerbeck and Christoph Bartneck. 2010. Perception of affect elicited by robot motion. In Proceedings of the 5th ACM/IEEE International Conference on Human-Robot Interaction (HRI'10). ACM, New York, NY, 53-60.

[40] Margrit Schreier. 2014. Qualitative content analysis. In The SAGE Handbook of Qualitative Data Analysis, Uwe Flick (Ed.). Sage Publications, Los Angeles, CA, 170-183.

[41] Megha Sharma, Dale Hildebrandt, Gem Newman, James E. Young, and Rasit Eskicioglu. 2013. Communicating affect via flight path: Exploring use of the Laban effort system for designing affective locomotion paths. In Proceedings of the 8th ACM/IEEE International Conference on Human-Robot Interaction (HRI'13). IEEE Press, Piscataway, NJ, 293-300.

[42] David Sirkin, Brian Mok, Stephen Yang, and Wendy Ju. 2015. Mechanical ottoman: How robotic furniture offers and withdraws support. In Proceedings of the 10th Annual ACM/IEEE International Conference on Human-Robot Interaction (HRI'15). ACM, New York, NY, 11-18. https://doi.org/10.1145/2696454.2696461

[43] Sichao Song and Seiji Yamada. 2017. Expressing emotions through color, sound, and vibration with an appearanceconstrained social robot. In Proceedings of the 2017 ACM/IEEE International Conference on Human-Robot Interaction (HRI'17). ACM, New York, NY, 2-11. https://doi.org/10.1145/2909824.3020239

[44] Viola Spolin and Paul Sills. 1999. Improvisation for the Theater: A Handbook of Teaching and Directing Techniques. Northwestern University Press, Evanston, IL.

[45] Leila Takayama, Doug Dooley, and Wendy Ju. 2011. Expressing thought: Improving robot readability with animation principles. In Proceedings of the 2011 6th ACM/IEEE International Conference on Human-Robot Interaction (HRI'11). IEEE Press, Piscataway, NJ, 69-76. https://doi.org/10.1145/1957656.1957674

[46] Larissa Z. Tiedens and Alison R. Fragale. 2003. Power moves: complementarity in dominant and submissive nonverbal behavior. F. Pers. Soc. Psychol. 84, 3 (2003), 558-568. 
[47] Florian Vaussard, Michael Bonani, Philippe Rétornaz, Alcherio Martinoli, and Francesco Mondada. 2011. Towards autonomous energy-wise RObjects. In Conference Towards Autonomous Robotic Systems (TAROS'11). Springer, Berlin, 311-322.

[48] Michael Walker, Hooman Hedayati, Jennifer Lee, and Daniel Szafir. 2018. Communicating robot motion intent with augmented reality. In Proceedings of the 2018 ACM/IEEE International Conference on Human-Robot Interaction (HRI'18). ACM, New York, NY, 316-324. https://doi.org/10.1145/3171221.3171253

[49] Yuto Yamaji, Taisuke Miyake, Yuta Yoshiike, P. Ravinda S. De Silva, and Michio Okada. 2011. STB: Child-dependent sociable trash box. Int. J. Soc. Robot. 3, 4 (2011), 359-370. https://doi.org/10.1007/s12369-011-0114-y

[50] Stephen Yang, Brian Mok, David Sirkin, Hillary Ive, Rohan Maheshwari, Kerstin Fischer, and Wendy Ju. 2015. Experiences developing socially acceptable interactions for a robotic trash barrel. In Proceedings of the 2015 24th IEEE International Symposium on Robot and Human Interactive Communication (RO-MAN'15). IEEE Press, Piscataway, NJ, 277-284. https://doi.org/10.1109/ROMAN.2015.7333693

[51] Genta Yoshioka, Takafumi Sakamoto, and Yugo Takeuchi. 2015. Inferring affective states from observation of a robot's simple movements. In Proceedings of the 2015 24th IEEE International Symposium on Robot and Human Interactive Communication (RO-MAN'15). IEEE Press, Piscataway, NJ, 185-190. https://doi.org/10.1109/ROMAN.2015.7333582

[52] James Young, Ehud Sharlin, and Takeo Igarashi. 2013. Teaching robots style: designing and evaluating style-bydemonstration for interactive robotic locomotion. Hum.-Comput. Interact. 28, 5 (2013), 379-416. https://doi.org/10. 1080/07370024.2012.697046

Received March 2018; revised December 2018; accepted January 2019 